Document downloaded from:

http://hdl.handle.net/10251/58237

This paper must be cited as:

Baselga Moreno, S.; García-Asenjo Villamayor, L.; Garrigues Talens, P. (2014).

Submillimetric GPS distance measurement over short baselines: noise mitigation by global robust estimation. Measurement Science and Technology. 25(10):1-6. doi:10.1088/09570233/25/10/105004.

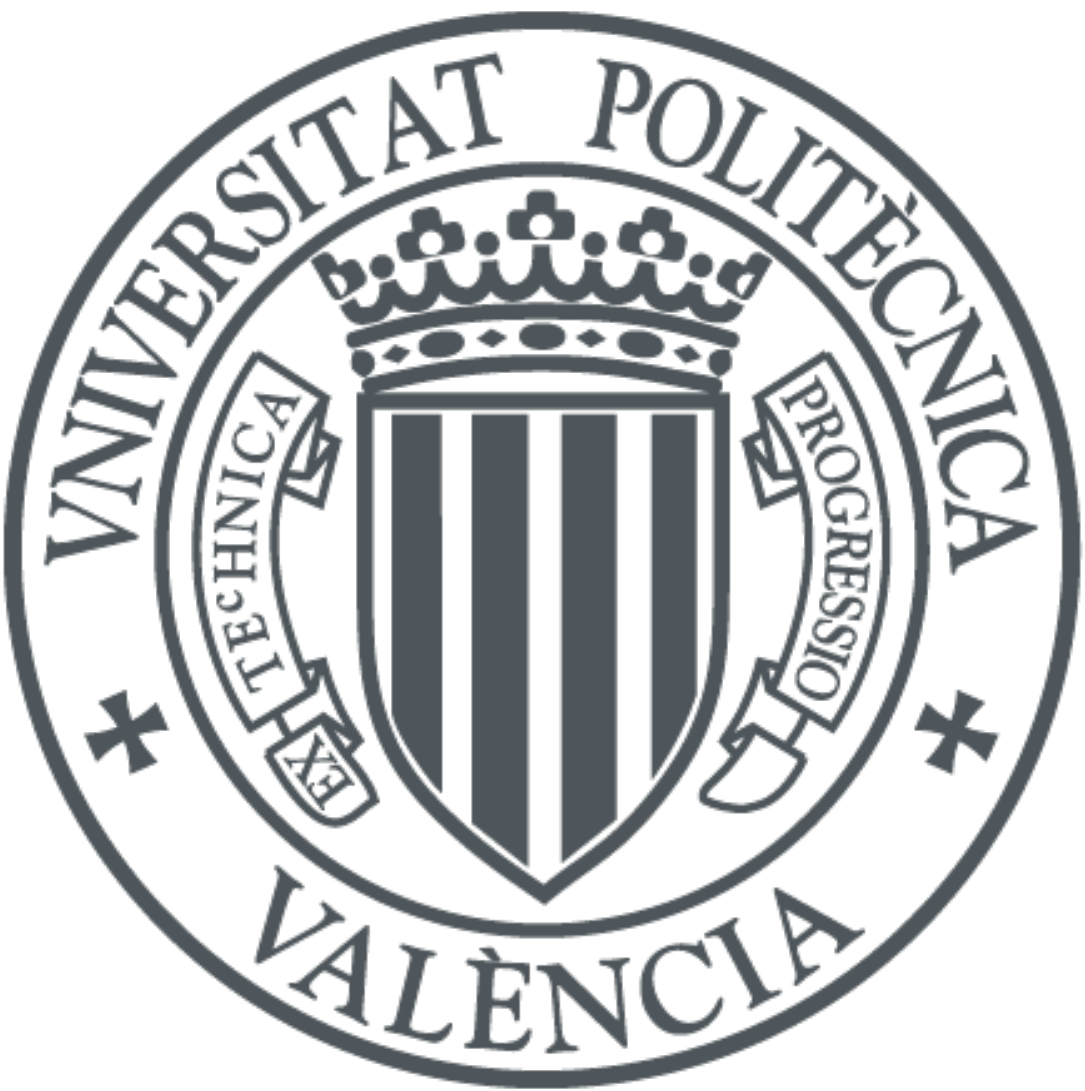

The final publication is available at

http://dx.doi.org/10.1088/0957-0233/25/10/105004

Copyright IOP Publishing: Hybrid Open Access

Additional Information 


\title{
Submillimetric GPS distance measurement over short baselines: noise mitigation by Global Robust Estimation
}

\author{
S Baselga, L García-Asenjo and P Garrigues \\ Dpto. Ing. Cartográfica, Geodesia y Fotogrametría, Universidad Politécnica de Valencia, \\ Camino de Vera s/n, 46022 Valencia, Spain \\ E-mail: serbamo@cgf.upv.es (S. Baselga)
}

\begin{abstract}
The potential use of GPS for precise length determination is currently a topic of extensive research. A prior work was dedicated to submillimetric length determination over short baselines under ideal conditions of data availability and clear environment. This paper presents a new computation method suited to the less favourable working conditions that are usually encountered in practice, which is based on both robust estimation theory and the use of an ambiguity free estimation method. As the experimental comparisons with the standard procedure based on least squares ambiguity determination show, it provides more stable values and permits to obtain results significant to the submillimetre level with time spans of the order of a few hours.
\end{abstract}

Keywords: distance measurement, short baseline, submillimetric accuracy, GPS, robust estimation

\section{Introduction}

Distance measurement with submillimetre accuracy in the open air for distances from a few meters to a few hundred meters is a challenging task at the present moment. Apart from the traditional use of submillimetric electronic distancemeters (EDM), there has recently been a growing interest in the potential use of GPS technology for precise length determination (Koivula et al 2012).

EDM length determination with submillimetre accuracy is a complicated task since it requires, first, accurate knowledge of the index of refraction of the propagation medium, which involves accurate determination of the existing conditions of atmospheric pressure, temperature and humidity and, second, the costly task of a prior calibration of the EDM in a dedicated infrastructure that provides traceability to the SI meter.

Conversely, GPS scale is acknowledged to be highly accurate for geodetic purposes, i.e. known at the level of $1 \mathrm{ppb}\left(10^{-9}\right)$ for worldwide scale, since it is based on satellite precise orbits that are currently known to the $1 \mathrm{~cm}$ accuracy level. Its scale stability for short distance determination, however, is not so clear. We dedicated a previous work to the study of GPS reproducibility, i.e. the inner consistency of GPS measurements, in short baselines and we found that the standard positioning procedure (coordinate determination after ambiguity fixation using $\mathrm{L}_{1}$ carrier phase observables) for $1 \mathrm{~h}$ time span baselines yields lengths that vary within an interval of a few millimetres along the day (Baselga et al 2013). It was also shown that submillimetric precision results can be obtained after some hours of observation - ideally $24 \mathrm{~h}$ and using the same equipment in both baseline ends - and that these results are significantly consistent in the long term (along the year) despite the existence of small systematic effects of the order of a few tenths of a millimetre.

In the present paper we want to benefit from a couple of tools, namely global robust estimation and an ambiguity-free functional model, to propose a new strategy for length determination that considerably reduces noise amplitude and permits to obtain a reliable solution with less observation time spans whereas being comparable in terms of computation time to the standard least squares ambiguity determination approach.

The motivation for our approach can be founded on the following three bases. 
First, there may be non-Gaussian errors in GPS measurements or, equivalently as Niu et al (2013) concluded, "it is not always optimal to model the GNSS positioning noise as white noise". That is, some systematic or gross errors may appear: most noticeably, as we experienced in Baselga et al (2013), a sidereal period signal possibly due to multipath, which is known to play a major role in short baselines (Amiri-Simkooei and Tiberius 2007). Besides, as Altamimi et al (2011) noted, seasonal signals may be found in GPS, since, e.g., the Earth is continuously deforming due to nontidal loading effects but modelling of these phenomena is still not incorporated in the processing of geodetic data by the IERS Technique Centres. According to Griffits and Ray (2012) orbit mismodelling or deficiencies in EOP tide models may also be expected. And finally, errors or approximations in the antenna or radome calibration may be expected (Ray et al 2008), especially since individual antenna calibrations are affected at the few millimetre level by near field multipath (Baire et al 2013).

Second, Robust estimation (RE) techniques were developed to obtain a solution that is maximum resistant towards the influence of gross and systematic errors (Andrews et al 1972, Huber 1981). They have been extensively used in geodetic and surveying problems (Fuchs 1982, Chen et al 1987, Harvey 1993, Yang 1999, Yang et al 2002, etc.) and, in particular, successfully applied to GPS positioning after the pioneer work by Wieser and Brunner (2002). In Baselga (2007) it was shown that a robust estimator has to be computed as a global optimization problem in order to be truly robust; otherwise it may result in a local optimum rather than the global one. This strategy, named Global Robust Estimation (GRE), was then applied to the successful determination of single frequency baselines affected by strong ionospheric delays that were intractable by the classical least squares approach (Baselga and García-Asenjo 2008a) and multipath mitigation (Baselga and García-Asenjo 2008b).

Third, our present problem is the determination of a short baseline with submillimetric accuracy in real working conditions. Knowledge of initial approximate coordinates of around 1 or $2 \mathrm{~cm}$ accuracy is here regarded as a prerequisite, which can be trivially obtained by standard GPS positioning (or PPP). Once these coordinates are known there is no longer need for the ambiguity resolution determination approach and the ambiguity-free functional model in Baselga (2014) can be used instead. Searching in the coordinate domain instead of the coordinate plus ambiguity domain dramatically reduces the search space dimension to three, thus enabling a quick search by the global optimization method by which the robust estimator is to be computed. The explanation of these second and third bases, i.e. GRE and the proposed ambiguity-free method, is the subject of the next section of the paper.

As a final remark we must acknowledge that the final step for a successful theory of submillimetric GPS length determination has to be a comprehensive study of compatibility with length patterns traced to the definition of the meter (absolute scale). Highly precise reproducibility, as addressed in the present paper, is merely a necessary though not sufficient condition for precise accuracy, which includes closeness to the absolute meter. In the Experiments section we include the comparison of a distance derived both by GPS and by an EDM traced to the SI meter. We understand that the successful comparison in one baseline - or half a dozen baselines - does not suppose an experimental demonstration of compatibility, but again it has to be fulfilled as a necessary condition. Extensive experimental comparisons are required to fully verify this compatibility. At any rate, the fact that the scale agreement found among GPS and the other space geodetic techniques is better than $1.10^{-9}$ (Altamimi et al 2011) makes us regard the possibility of absolute submillimetric GPS distance determination for lengths of some hundred metres as a plausible assumption worth of thorough verification.

\section{Functional model and solution}

Let us write the double-differenced carrier phase observation equation for receivers $i$ and $j$ and satellites $k$ and $l$ as

$$
\lambda \phi_{i j}^{k l}=\rho_{i j}^{k l}+\lambda N_{i j}^{k l}+\varepsilon_{i j}^{k l}
$$


where $\lambda$ is the carrier wavelength, and $\phi_{i j}^{k l}, \rho_{i j}^{k l}$, and $N_{i j}^{k l}$ are respectively the double-differenced carrier phases, ranges and integer ambiguities for subtractions ordered as follows

$$
\phi_{i j}^{k l}=\left(\phi_{j}^{l}-\phi_{i}^{l}\right)-\left(\phi_{j}^{k}-\phi_{i}^{k}\right)
$$

For sufficiently short baselines it is assumed that ionospheric and tropospheric delays, instrumental delays and clock errors are cancelled, thus resulting in a zero-centred Gaussian double-differenced residual $\varepsilon_{i j}^{k l}$. However, it has to be emphasized, this is not the case if multipath or any other periodic effect plays a non-negligible role, such as in the case of submillimetric length determination, as it was shown in Baselga et al (2013). Being only interested in the case of short baselines the use of only L1 frequency observables is unquestionable, since they are less noisy than L2 observables or any linear combination of observables (Xu 2003).

The use of the least-squares estimator in a functional model, Eq. (1), where residuals are not zerocentred Gaussian variables provides suboptimal results and a robust estimator must be preferred instead (Huber 1981). Considering that a robust estimator is optimally computed as a global optimization problem and that the minimum $\mathrm{L}_{1}$-norm (please note that there is a conflict of notation here, $\mathrm{L}_{1}$-norm refers to the minimum sum of absolute residuals and has not to be confused with the GPS frequency $L_{1}$ ) has been shown to be a successful robust estimator (e.g. Fuchs 1982, Harvey 1993, Baselga and García-Asenjo 2008a, 2008b) we will solve for the coordinates implicit in (1) after a few manipulations along with the statistic condition of minimum $\mathrm{L}_{1}$-norm.

We can arrange (1) as

$$
\phi_{i j}^{k l}-\frac{\rho_{i j}^{k l}}{\lambda}=N_{i j}^{k l}+\frac{\varepsilon_{i j}^{k l}}{\lambda}
$$

and have on the right side an integer value $N_{i j}^{k l}$ plus a small fractional value $\varepsilon_{i j}^{k l} / \lambda$ since the noise is of the order of millimetre and $\lambda$ is of the order of decimetre $\left(19 \mathrm{~cm}\right.$ for GPS $\left.\mathrm{L}_{1}\right)$. Denoting by Int( ) the operation of rounding to the nearest integer we can have a rather simple model (Baselga 2014)

$$
\phi_{i j}^{k l}-\frac{\rho_{i j}^{k l}}{\lambda}-\operatorname{Int}\left(\phi_{i j}^{k l}-\frac{\rho_{i j}^{k l}}{\lambda}\right)=\varepsilon_{i j}^{\prime k l}
$$

where $\varepsilon_{i j}^{k l}=\varepsilon_{i j}^{k l} / \lambda$ is a unitless residual. One normally fixes one baseline end, say $i$, and solves for the coordinates of the other, $j$, that are implicit in $\rho_{i j}^{k l}$. The usual procedure involves linear expansion of $\rho_{i j}^{k l}$ around the approximate coordinates for $j$ and determination of the differential corrections to them. Since we are proposing to use a global optimization method this step is unnecessary and we will directly search for the optimum coordinates $\left(X_{j}, Y_{j}, Z_{j}\right)$ implicit in $\rho_{i j}^{k l}$ on the first side.

This functional model (4) has to be complemented with a minimum condition in order to be solved. As said, the assumption of zero-centred Gaussian residuals that leads to the least-squares estimator is not the best option when there may be systematic effects or gross errors. A much more robust estimator is the minimum $\mathrm{L}_{1}$-norm estimator derived from the Laplace or double exponential distribution, which is a symmetric distribution that decays from the mean much faster than the Gaussian distribution. If $\varepsilon_{i j}^{k l}$ are the observation residuals, the minimum $\mathrm{L}_{1}$-norm estimator is written 


$$
\sum_{\text {obs }}\left|\varepsilon_{i j}^{k l}\right| \rightarrow \min
$$

where the summation is done for all observation residuals. Considering our functional model (4) we can now state our global optimization problem as

$$
\left\{\begin{array}{l}
\min f \\
\operatorname{being} f\left(X_{j}, Y_{j}, Z_{j}\right)=\sum_{\text {obs }}\left|\phi_{i j}^{k l}-\frac{\rho_{i j}^{k l}}{\lambda}-\operatorname{Int}\left(\phi_{i j}^{k l}-\frac{\rho_{i j}^{k l}}{\lambda}\right)\right| \\
\operatorname{for}\left(X_{j}, Y_{j}, Z_{j}\right) \in D
\end{array}\right.
$$

where the search domain $D$ can be defined, for instance, as the cube centred in the initial approximate coordinates $\left(X_{j 0}, Y_{j 0}, Z_{j 0}\right)$ with corners in $\left(X_{j 0} \pm \varepsilon, Y_{j 0} \pm \varepsilon, Z_{j 0} \pm \varepsilon\right)$ being $\varepsilon$ a sensible figure for the correct solution to be included in the domain, e.g. $1 \mathrm{~cm}$ if this complies with the existing degree of accuracy of the approximate coordinates.

We could thought of each of these $2 \mathrm{~cm}$-length cube sides to be divided into parts of $0.1 \mathrm{~mm}$ and thus have a three-dimensional lattice of $201^{3} \approx 8 \times 10^{6}$ possible solutions. Each of the feasible solutions could be plugged into the objective function $f$ in (6) and retain the one with a minimum value for $f$ as the solution to our problem. This brute-force procedure can be easily done by a computer in a few seconds or even less (depending on the number of observations), but it is advisable to perform the search in a more sensible manner and follow one of the established global optimization methods, like the simulated annealing (SA) method. For further details on SA the reader is referred to Baselga (2010) and references therein.

Once the unknown coordinates $\left(X_{j}, Y_{j}, Z_{j}\right)$ have been determined, the baseline length is straightforwardly obtained

$$
L=\sqrt{\left(X_{j}-X_{i}\right)^{2}+\left(Y_{j}-Y_{i}\right)^{2}+\left(Z_{j}-Z_{i}\right)^{2}}
$$

It is worth mentioning that the computation of coordinates and subsequent derivation of the distance remains up to the moment as the most consistent method for the determination of the baseline length. Other functional models may be established directly based on the baseline length as an unknown, but, to our knowledge, they all yield poorer results in terms of precision.

Once the rationale for our new strategy and the corresponding solution method have been introduced, we present in the following sections applications to real data where the comparison between the traditional solution and the proposed strategy permits us to conclude on the degree of improvement attained.

\section{Experiments}

\subsection{Experiment 1}

We start by applying the new strategy to some representative data of our previous work (Baselga et al 2013) where baseline lengths were obtained after positioning by traditional least squares ambiguity determination. In particular, we will focus on the baseline LNC1-LNC2, see features in Table 1, whose stations belong to CORS network (Snay and Soler 2008).

Table 1. Baseline features for experiment 1. 


\begin{tabular}{ccccccc}
\hline $\begin{array}{c}\text { Baseline } \\
\text { (network) }\end{array}$ & $\begin{array}{c}\text { Length } \\
(\mathrm{m})\end{array}$ & $\begin{array}{c}\text { Azimuth } \\
\text { (gon) }\end{array}$ & $\begin{array}{c}\text { Approx. } \\
\text { lat } / \text { lon }\end{array}$ & Antennas (radomes) & $\begin{array}{c}\text { Available } \\
\text { calibration }\end{array}$ & Monuments \\
\hline $\begin{array}{c}\text { LNC1 } \\
\text { LNC2 }\end{array}$ & 27.326 & 299.17 & $39^{\circ} /-121^{\circ}$ & 2 ASH701945E_M & f(az,elev) & $\begin{array}{c}\text { Concrete } \\
\text { pillars }\end{array}$ \\
\hline
\end{tabular}

By using IGS final ephemerides (Dow et al 2009), the most recent igs08.atx absolute antenna calibration file and an elevation mask of $15^{\circ}$ we formed double-differenced $\mathrm{L}_{1}$-carrier phases and obtained solutions both by the traditional method based on least squares ambiguity determination and by the new strategy of GRE with the ambiguity-free model.

\subsection{Experiment 2}

We also want to analyze the performance of both methods under more common field working conditions, in particular, for baselines that are not being continuously measured but only occupied for a period of a few hours. Therefore we used a pair of Leica AR25 choke ring antennas, with the corresponding signal splitters and four Trimble 5700 receivers to observe during $6 \mathrm{~h}$ on a pair of geodetic pillars belonging to the Universidad Politécnica de Valencia (UPV) campus EDM calibration baseline, Table 2. IGS ephemerides and absolute antenna calibration were also used, and we obtained solutions by both methods for different observation time spans.

Table 2. Baseline features for experiment 2.

\begin{tabular}{ccccccc}
\hline Baseline & $\begin{array}{c}\text { Length } \\
(\mathrm{m})\end{array}$ & $\begin{array}{c}\text { Azimuth } \\
\text { (gon) }\end{array}$ & $\begin{array}{c}\text { Approx. } \\
\text { lat / lon }\end{array}$ & Antennas (radomes) & $\begin{array}{c}\text { Available } \\
\text { calibration }\end{array}$ & Monuments \\
\hline $\begin{array}{c}\text { UPV } \\
\text { campus }\end{array}$ & 94.401 & 332.84 & $39^{\circ} / 0^{\circ}$ & $\begin{array}{c}\text { 2 LEIAR25 } \\
\text { (SCIT) }\end{array}$ & f(az,elev) & $\begin{array}{c}\text { Concrete } \\
\text { pillars }\end{array}$ \\
\hline
\end{tabular}

\section{Results and discussion}

When we compute distances by the standard approach, i.e. after least squares adjustment ambiguity determination, we observe the typical length variations in a range of a few millimetres along a day. By contrast, the proposed approach of GRE with an ambiguity-free functional model provides more stable solutions. Figure 1 shows the 24 distances obtained for a typical day - in particular, day 263 of year 2011 - for baseline LNC1-LNC2, where each distance has been computed from a $1 \mathrm{~h}$ observation time span.

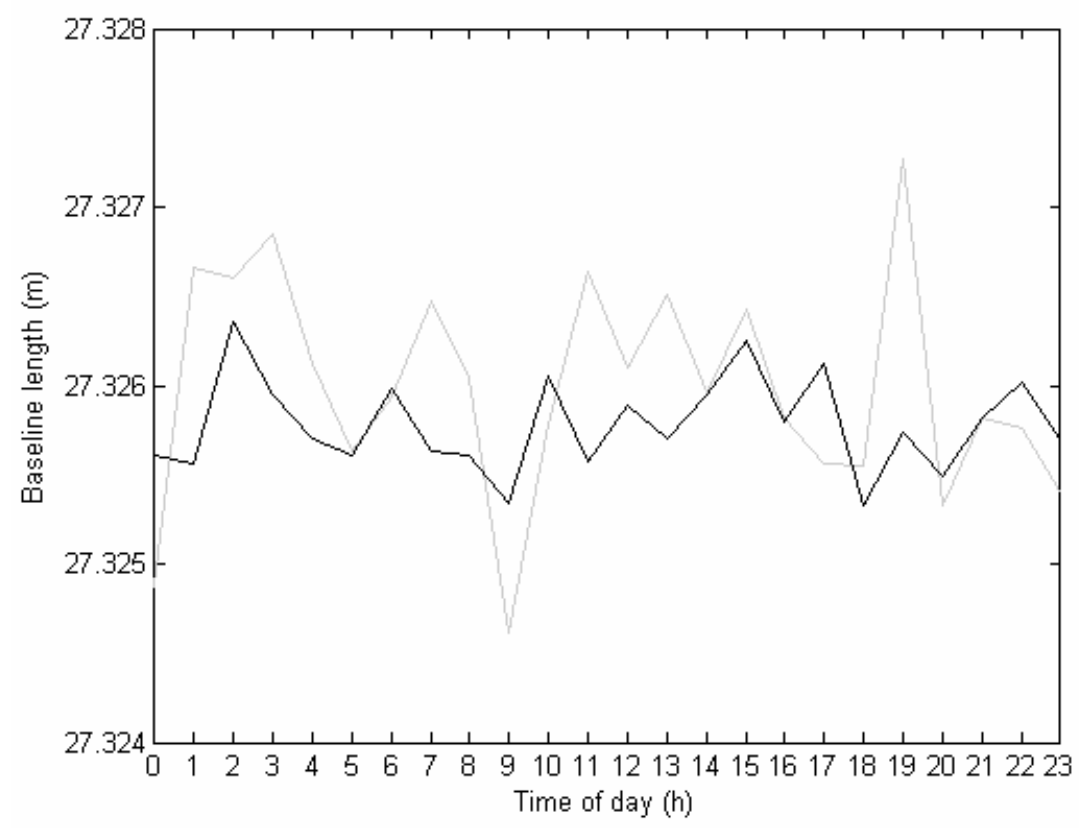


Figure 1. LNC1 - LNC2 baseline length values for day 263 by the standard approach (grey line) and GRE (black line).

Figure 2 shows baseline length results along one week. As it can be seen, this variation within a range below one millimetre is maintained for GRE. Table 3 summarizes the results obtained with both methods after processing $1 \mathrm{~h}$ time span vectors for the entire year. In particular, it is worth to note how the maximum deviation from the means decreases from $2.3 \mathrm{~mm}$ with the standard approach to $1.1 \mathrm{~mm}$ with the proposed method of GRE over the ambiguity-free model.

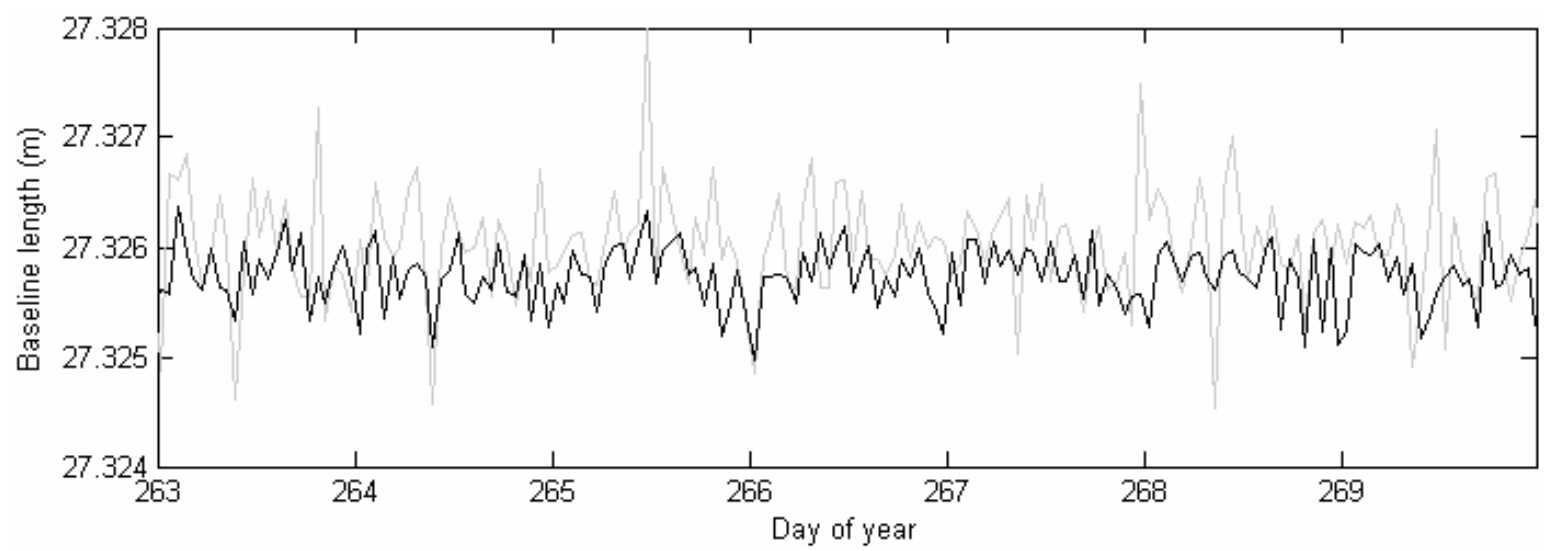

Figure 2. LNC1 - LNC2 baseline length values for one week by the standard approach (grey line) and GRE (black line).

Table 3. Statistics for $1 \mathrm{~h}$ time span vectors processing by both methods. Values in $\mathrm{m}$.

\begin{tabular}{|l|c|c|}
\hline & Standard & $\begin{array}{c}\text { GRE ambiguity- } \\
\text { free model }\end{array}$ \\
\hline Mean & 27.3258 & 27.3256 \\
\hline Median & 27.3258 & 27.3256 \\
\hline Standard deviation & 0.0005 & 0.0003 \\
\hline Maximum value & 27.3278 & 27.3263 \\
\hline Minimum value & 27.3235 & 27.3245 \\
\hline Maximum deviation from the mean & 0.0023 & 0.0011 \\
\hline
\end{tabular}

In view of all of these figures we may have, in general, the hope of obtaining results significant to the submillimetre level with short observation periods, possibly two or three hours, by using GRE and the ambiguity-free model.

Now we study the results obtained in our UPV baseline where the observation conditions were less favourable, especially with regard to potential reflections on the surrounding buildings and trees, as well as for the limitation in observation times to hours instead of months, what makes the experiment results more meaningful to reflect most real working conditions.

As it was mentioned, we used four receivers connected to the pair of signal splitters attached to each of the antennas. Thus we could first analyze the zero baseline observation residuals, exclusively due to the internal precision of the receivers. The double-differenced carrier phase 
residuals for the zero baselines were typically below $2 \mathrm{~mm}$, which represents undifferenced carrier phase residuals below $1 \mathrm{~mm}$.

A reference value for the measured length had been previously obtained by means of an absolute scale transfer campaign from the Nummela baseline - see Jokela and Häkli (2006) - with a Mekometer ME5000 EDM: $94.4010 \pm 0.0002(2 \sigma) \mathrm{m}$.

For the total of 6 hours of observation data we computed baselines for time spans of 30 minutes, 1 hour, 1 hour and 30 minutes, and 2 hours. For each experiment the corresponding baselines have been numbered and therefore we have 12 baselines in the first experiment, 6 in the second, 4 in the third and 3 in the forth. With time spans of 30 minutes - Figure 3a) - we can observe no significant differences between the two approaches: in both of them some of the computed baselines, especially the first ones, differ some millimetres from the reference value. The situation seems not to improve much with time spans of 1 hour, Figure 3b). A careful look, however, must lead us to consider that with the exception of the second value all lengths are within $1 \mathrm{~mm}$ of the reference value for the case of the GRE method, or $3 \mathrm{~mm}$ for the standard method. As a plausible hypothesis one may regard the existing multipath environment as the responsible for these quite large figures that are intractable even for the GRE method. For the 1 hour and 30 minutes time spans both methods result in a solution were large amplitudes have been clearly reduced (Figure 3c): only one value remains around 1.5 millimetres from the reference solution for the case of the classic approach, whereas all four values are clearly within 1 millimetre for the GRE method. Finally, with 2 hour baselines both type of solutions are clearly within 1 millimetre from the reference value.
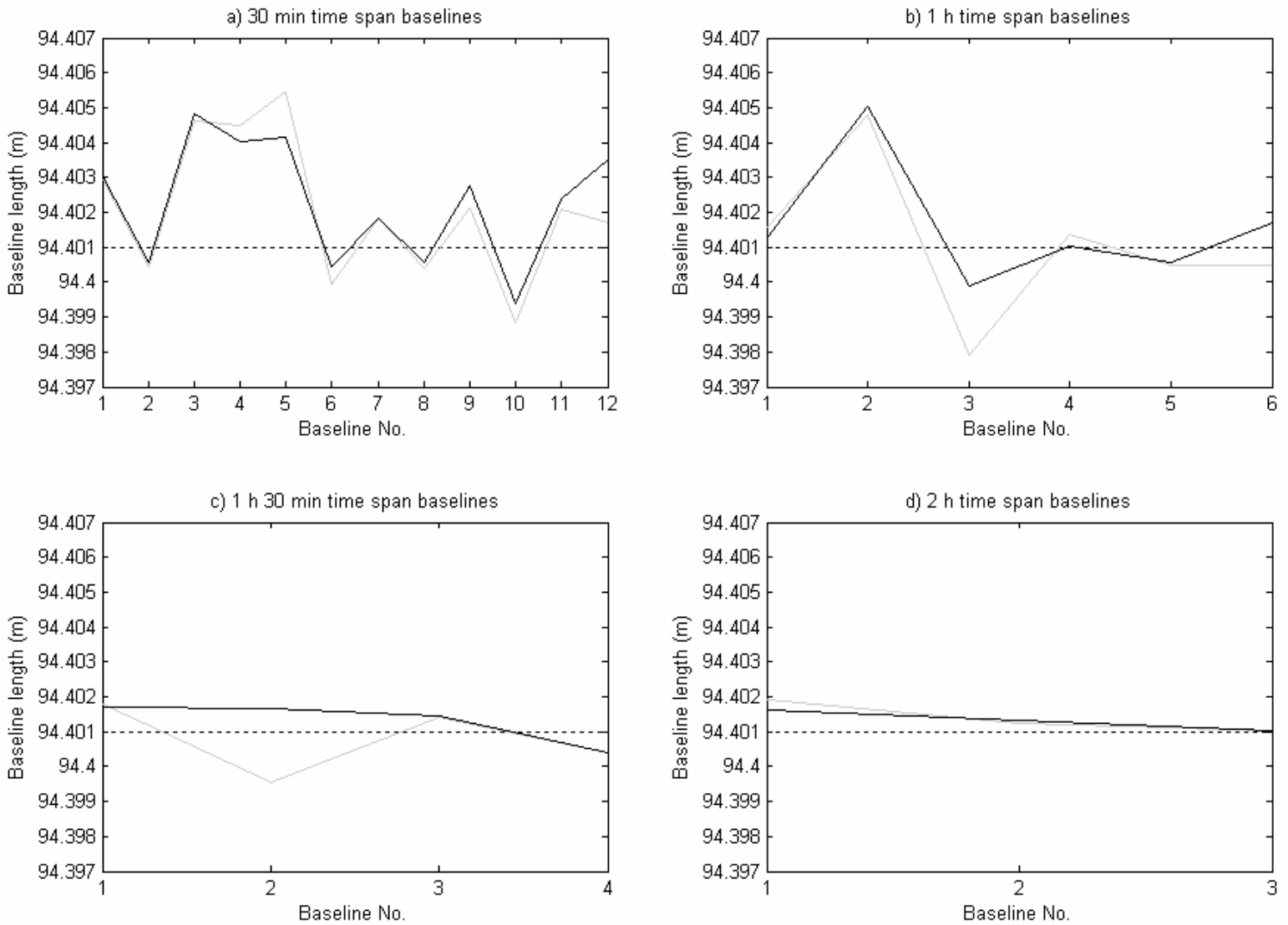

Figure 3. UPV baseline lengths obtained by the standard approach (grey line) and GRE (black line) compared with the reference value (dotted line) for different time spans: a) 30 minutes, b) 1 hour, c) 1 hour 30 minutes and d) 2 hours.

As a result of the two experiments, we can conclude that GPS length determination with significance up to the submillimetre level may be attainable after short observation periods - a few hours - by means of GRE with an ambiguity-free method. The classic length determination 
procedure after least squares ambiguity determination seems to show a lower performance although it can also yield submillimetric results with longer observation periods. In any case, we want to remember that, as we said before, extensive research is needed to confirm the GPS scale consistency with the absolute meter.

\section{Conclusion}

After a prior work in which GPS submillimetric distance determination over short baselines was studied under ideal conditions of both observation time duration and clear environment, a new computation method has been proposed bearing in mind the practical application to less favourable conditions.

The method is based on both robust estimation theory and the use of a previously presented ambiguity-free GPS functional model. As it is observed in experimental comparisons with the standard procedure based on least squares ambiguity determination it provides more stable values and permits to obtain meaningful results at the submillimetre level with shorter observation time spans of the order of a few hours.

More experimental field tests are needed however to confirm the degree of improvement of the procedure proposed in this paper as well as the compatibility of the obtained metre with the SI metre realized by other metrology techniques.

Acknowledgments This research is funded by the Spanish Ministry of Science and Innovation (AYA201123232).

\section{References}

Altamimi Z, Collilieux X and Métivier L 2011 ITRF2008: an improved solution of the international terrestrial reference frame $J$. Geod. 85 457-73

Amiri-Simkooei AR and Tiberius CCJM 2007 Assessing receiver noise using GPS short baseline time series GPS Solut. 11 21-35

Andrews D F, Bickel P J, Hampel F R, Huber P J, Rogers W H and Tukey J W 1972 Robust estimates of location: Surveys and advances (Princeton, N.J.: Princeton University Press)

Baire Q, Bruyninx C, Legrand J, Pottiaux E, Aerts W, Defraigne P, Bergeot N and Chevalier JM 2013 Influence of different GPS receiver antenna calibration models on geodetic positioning GPS Solut. doi: 10.1007/s10291-013-0349-1

Baselga S 2007 Global Optimization Solution of Robust Estimation J. Surv. Eng. 133 123-8

Baselga S 2010 Global optimization applied to GPS positioning by ambiguity functions Meas. Sci. Technol. 21125102

Baselga S 2014 Ambiguity-Free Method for Fast and Precise GNSS Differential Positioning J. Surv. Eng. $14022-7$

Baselga S and García-Asenjo L 2008a GNSS Differential Positioning by Robust Estimation J. Surv. Eng. 134 21-5

Baselga S and García-Asenjo L 2008b Multipath Mitigation by Global Robust Estimation J. Navig. 61 1-8

Baselga S, García-Asenjo L and Garrigues P 2013 Submillimetric GPS distance measurement over short baselines: case study in inner consistency Meas. Sci. Technol. 24075001

Chen Y Q, Kavouras M and Chrzanowski A 1987 A strategy for the detection of outlying observations in measurements of high precision Can. Surveyor 41 527-40

Dow JM, Neilan RE and Rizos C 2009 The International GNSS Service in a changing landscape of Global Navigation Satellite Systems J. Geod. 83 191-8

Fuchs H 1982 Contribution to the adjustment by minimizing the sum of absolute residuals Manuscr. Geod. 7 $151-207$ 
Griffits J and Ray JR 2012 Sub-daily alias and draconitic errors in the IGS orbits GPS Solut. doi: 10.1007/s10291-012-0289-1

Harvey B R 1993 Survey network adjustments by the L1 method Austral. J. Geod. Photogram. Survey. 59 39-52

Huber P J 1981 Robust statistics (New York: Wiley)

Jokela J and Häkli P 2006 Current research and development at the Nummela standard baseline. 23rd FIG Congres ( Munich, Germany, 8-13 October) PS5.2.2 pp 1-15

Koivula H, Häkli P, Jokela J, Buga A and Putrimas R 2012 GPS metrology: bringing traceable scale to local crustal deformation network Geodesy for Planet Earth (Int. Association of Geodesy Symp. vol 136) ed S

Niu X, Chen Q, Zhang Q, Zhang H, Niu J, Chen K, Shi C and Liu J 2013 Using Allan variance to analyze the error characteristics of GNSS positioning GPS Solut. doi 10.1007/s10291-013-0324-x

Ray J, Altamimi Z, Collilieux X and van Dam T 2008 Anomalous harmonics in the spectra of GPS position estimates GPS Solut. 12 55-64

Snay R A and Soler T 2008 Continuously Operating Reference Station (CORS): History, Applications, and Future Enhancements J. Surv. Eng. 134 95-104

Wieser A and Brunner F K 2002 Short static GPS sessions: Robust estimation. GPS Solut. 5 70-79

Xu G 2003 GPS: Theory, Algorithms and Applications (Berlin Heidelberg: Springer-Verlag)

Yang Y 1999 Robust estimation of geodetic datum transformation J. Geod. 73 268-74

Yang Y, Song L and Xu T 2002 Robust estimator for correlated observations based on bifactor equivalent weights J. Geod. 76 353-8 